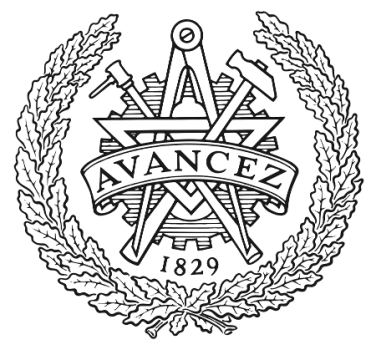

CHALMERS

UNIVERSITY OF TECHNOLOGY

\title{
Physiological recruitment of motor units by high-frequency electrical stimulation of afferent pathways
}

Downloaded from: https://research.chalmers.se, 2023-04-26 08:03 UTC

Citation for the original published paper (version of record):

Dideriksen, J., Muceli, S., Dosen, S. et al (2015). Physiological recruitment of motor units by high-frequency electrical stimulation of afferent pathways. Journal of Applied Physiology, 118(3): 365-376.

http://dx.doi.org/10.1152/japplphysiol.00327.2014

N.B. When citing this work, cite the original published paper. 


\title{
Physiological recruitment of motor units by high-frequency electrical stimulation of afferent pathways
}

\author{
Jakob L. Dideriksen, Silvia Muceli, Strahinja Dosen, Christopher M. Laine, and Dario Farina \\ Department of Neurorehabilitation Engineering, Bernstein Focus Neurotechnology Göttingen, Bernstein Center \\ for Computational Neuroscience, University Medical Center Göttingen, Georg-August University, Göttingen, Germany
}

Submitted 10 April 2014; accepted in final form 30 November 2014

\begin{abstract}
Dideriksen JL, Muceli S, Dosen S, Laine CM, Farina D. Physiological recruitment of motor units by high-frequency electrical stimulation of afferent pathways. J Appl Physiol 118: 365-376, 2015. First published December 4, 2014; doi:10.1152/japplphysiol.00327.2014.-Neuromuscular electrical stimulation (NMES) is commonly used in rehabilitation, but electrically evoked muscle activation is in several ways different from voluntary muscle contractions. These differences lead to challenges in the use of NMES for restoring muscle function. We investigated the use of low-current, high-frequency nerve stimulation to activate the muscle via the spinal motoneuron (MN) pool to achieve more natural activation patterns. Using a novel stimulation protocol, the H-reflex responses to individual stimuli in a train of stimulation pulses at 100 $\mathrm{Hz}$ were reliably estimated with surface EMG during low-level contractions. Furthermore, single motor unit recruitment by afferent stimulation was analyzed with intramuscular EMG. The results showed that substantially elevated H-reflex responses were obtained during $100-\mathrm{Hz}$ stimulation with respect to a lower stimulation frequency. Furthermore, motor unit recruitment using $100-\mathrm{Hz}$ stimulation was not fully synchronized, as it occurs in classic NMES, and the discharge rates differed among motor units because each unit was activated only after a specific number of stimuli. The most likely mechanism behind these observations is the temporal summation of subthreshold excitatory postsynaptic potentials from Ia fibers to the MNs. These findings and their interpretation were also verified by a realistic simulation model of afferent stimulation of a MN population. These results suggest that the proposed stimulation strategy may allow generation of considerable levels of muscle activation by motor unit recruitment that resembles the physiological conditions.
\end{abstract}

neuromuscular electrical stimulation; H-reflex; motoneuron; electromyography; computational modeling

NEUROMUSCULAR ELECTRICAL STIMULATION (NMES) is a common tool for a wide range of applications in research and clinical settings to generate contractions in muscles whose voluntary activation is limited or absent $(12,56)$. The muscle activation is typically achieved via transcutaneous stimulation of motor axons, either at locations where the nerve innervating the muscle is sufficiently superficial, or above the muscle belly to stimulate the terminal axonal branches. A well-known limitation of NMES is that the characteristics of muscle contraction it induces are very different from voluntary contractions. For example, the size-ordered recruitment of motor units is not present, motor units are activated in perfect synchrony, and the elicited motor unit discharge rates are often high relative to their physiological distribution, since they are imposed by the

\footnotetext{
Address for reprint requests and other correspondence: D. Farina, Dept. of Neurorehabilitation Engineering, Bernstein Focus Neurotechnology Göttingen, Bernstein Center for Computational Neuroscience, Univ. Medical Center Göttingen, Georg-August Univ., Von Siebold-Str. 6, 37075 Göttingen, Germany (e-mail: dario.farina@bccn.uni-goettingen.de).
}

stimulation frequency. These differences determine several challenges in practical uses of NMES $(12,56)$.

Different strategies have been proposed to overcome these limitations. Some approaches rely on particular stimulation techniques, such as the application of ramp prepulses to achieve orderly recruitment of motoneurons (MNs) (42) or distributed stimulation to decrease the rate of development of muscle fatigue (57). Another proposed methodology relies on activation of MNs via the spinal cord using stimulation of afferent fibers at currents below activation of efferent fibers (11). This method is based on the rationale that activating the muscle via the spinal cord presumably evokes more natural muscle activation than direct motor fiber stimulation. Afferent fibers can be activated by electrical stimulation at peripheral sites where it would elicit recruitment of multiple types of nerves, including Ia, Ib, and cutaneous afferents (type II) projecting mono- and/or polysynaptically to the MNs $(50,65)$. Input from cutaneous afferents may either excite or inhibit MNs (38), possibly through modulation of Ia presynaptic inhibition (2), and $\mathrm{Ib}$ input elicits polysynaptic inhibition (32, 72). The primary afferent pathway delivering excitatory input to MNs is the Ia monosynaptic pathway, as indicated by the H-reflex $(50,65)$. In voluntary contractions, the Ia pathway conveys information from muscle stretch receptors and contributes significantly to the net excitation of the MN pool (55). The level of muscle activation that can be evoked by delivering an electrical stimulus in this way, however, is lower than the maximum muscle activation evoked by motor axon stimulation (M-wave) (75), in particular when stimulation occurs over the muscle belly and not at the nerve bundle $(10,11)$. Targeted muscle activation via transcutaneous nerve bundle stimulation, however, is not possible for all muscles. This limitation, along with the limited range of contraction levels, implies that this approach is not suitable for all applications of NMES. To overcome these limitations, it has been suggested that activation of persistent inward currents (PIC) via brief periods of high-frequency stimulation of afferent fibers (14, 23) may increase the MN excitability and thereby amplify the H-reflex amplitude and elicit spontaneous asynchronous MN activity $(10,22)$. However, such centrally evoked amplification of the motor output cannot be elicited in all muscles (11), and the amplitude and duration of the evoked contractions are highly variable (29) and thus difficult to control.

In this study, we investigated the frequency dependence of $\mathrm{MN}$ recruitment through activation of afferent nerves (presumably predominantly via the Ia pathway) and its underlying mechanisms. It is well known that the magnitude of the response of the MNs to electrically induced neural input depends on the background excitation in the MN population (75). A relatively low input will evoke a larger response if the 
excitability of many MNs is near the threshold for generation of action potentials. The excitatory postsynaptic potential (EPSP) induced by a Ia fiber action potential declines exponentially with a time constant between 4 and $12 \mathrm{~ms}$, as estimated for single-axon stimulation in cats (36). In humans, the duration of a compound Ia EPSP has been estimated to be $\sim 15$ ms $(6,71,80)$. This implies that temporal summation of subthreshold EPSPs from Ia fibers could lead to MN activation, provided that the stimulation frequency is sufficiently high, i.e., interstimulus interval shorter than the EPSP duration (53). This principle is supported by the observations that a light tendon tap delivered before an electrical stimulus increases its response within a brief time interval (46). Furthermore, it has been shown that the responses to two stimuli delivered within the duration of the Ia EPSP are produced by different groups of motor units, indicating that the MNs responding to the first stimulus were hyperpolarized at the time of the second one, while the MNs responding to the second stimulus could be activated only by the sum of two consecutive inputs (71).

The summation of EPSPs is explored in this study as a potential mechanism for functional NMES. If the MN excitability is progressively increased by EPSPs arriving with short intervals (high-frequency stimulation), it would be possible to obtain a muscle response for stimulation intensities that would not elicit any activation at baseline conditions (single stimulus). In the high-frequency condition, consecutive stimuli would generate $\mathrm{H}$-reflexes that represent the responses of different populations of MNs. Furthermore, the hypothesized mechanism of progressive increase in excitability by multiple stimuli would imply that MNs are activated only after a certain number of stimuli so that their activation frequency would be lower than the stimulation frequency. In this way, not all MNs would respond to the same stimuli, since each MN potentially requires a different number of prestimuli before its activation, so that the MNs would not be fully synchronized. This further suggests that MNs with higher excitability (low threshold) would require fewer stimuli per triggered action potential and thus would be driven at higher discharge rates compared with higher-threshold MNs, as in physiological conditions (54). Thus, according to this hypothesized recruitment mechanism, stimulating the afferent pathways at low intensity and high rate could generate a muscle activation with $\mathrm{MN}$ recruitment that resembles the physiological activation, i.e., physiological distribution of discharge rates, partly asynchronous discharges between MNs, potentially large number of active MNs, etc. To test this hypothesis, we investigated the H-reflex responses in the extensor carpi radialis longus muscle to single vs. multiple stimulation pulses delivered at different frequencies in the whole muscle and in single motor units, in both simulated and experimental data.

\section{METHODS}

Recruitment of MNs with high-frequency afferent stimulation was investigated by a computational model and in experimental conditions.

Computational model. A population of $180 \mathrm{MNs}$ was simulated as Hodgkin-Huxley type models, each consisting of one soma and one dendrite compartment (19). The membrane-specific capacitance was set to $1 \mu \mathrm{F}$, the axial resistivity to $70 \Omega \mathrm{cm}$, the soma specific resistance in the range from 1.15 to $0.65 \mathrm{k} \Omega \mathrm{cm}^{2}$, the dendrite specific resistance from 14.4 to $6.05 \mathrm{k} \Omega \mathrm{cm}^{2}$, and the equilibrium potential of the membrane and leakage voltage were set to $0 \mathrm{mV}$, while the equilibrium potentials were $120 \mathrm{mV}$ for the sodium and $-10 \mathrm{mV}$ for the potassium. Exponential distributions across the MN population were applied (37) within these ranges of the parameters (19). Innervation numbers (number of muscle fibers innervated by each $\mathrm{MN}$ ) were assumed proportional to the radius of the soma. A constant current was injected into the soma compartment of the neurons to simulate a constant supraspinal drive. Low-pass filtered $(<100 \mathrm{~Hz}$; Ref. 69) white noise was imposed on this current, for which the standard deviation was adjusted so that the coefficient of variation for the MN interspike intervals was between 10 and 30\% (21, 60, 67).

When an action potential occurred in an afferent fiber or in an interneuron, an excitatory postsynaptic current (EPSC) was injected into the soma compartment of the receiving neuron. This simplification [Ia boutons exist on the soma as well as on the dendrites (16)] was done since the EPSC shape was modeled based on recordings from the soma (36). The EPSC was modeled as an exponential decline with a time constant of $1.5 \mathrm{~ms}$ (36), unless stated otherwise (see below). Each Ia afferent fiber projected monosynaptically to all MNs (63, 70, 85). To represent variability in synaptic strengths $(51,63)$, each synaptic connection was randomly assigned a baseline peak EPSC selected from a log normal distribution with the mean and variance of $0.3 \mu \mathrm{A}$ and $1 \mu \mathrm{A}$, respectively.

Axon length was set to $1 \mathrm{~m}$, and the stimulation site was located 10 $\mathrm{cm}$ from the distal end. Axonal conduction velocity was randomly selected from a normal distribution (mean \pm SD: $105 \pm 20 \mathrm{~m} / \mathrm{s}$ ) for Ia fibers $(41,44,72)$, and individual velocity values were set according to an exponential distribution in the range $70-110 \mathrm{~m} / \mathrm{s}$ for motor axons $(8,26,41)($ mean $\pm \mathrm{SD}: 78 \pm 10 \mathrm{~m} / \mathrm{s})$. Axon rheobase current was set inversely proportional to the conduction velocity (73). In this way, the stimulation current required to evoke action potentials in each axon could be identified. The maximum stimulation current was defined as the current evoking action potentials in all Ia and motor axons. When activated, the action potential traveled in both directions of the axon from the stimulation site. $\mathrm{MN}$ action potentials were extinguished in case of antidromic collision.

In the first simulation paradigm, a descending drive of $1.5 \mathrm{nA}$ was imposed on the MN population. This level of excitation was approximately one-half of that required for stable recruitment of the smallest $\mathrm{MN}$. The H-reflex recruitment curves in response to one, two, or three stimuli at $100 \mathrm{~Hz}$ were simulated. For each number of stimuli, simulations were performed with $5-100 \%$ of the maximum stimulation intensity in increments of 5\%. All increments were performed in individual simulations, each of which was repeated five times to accommodate for the randomly assigned parameters in the model. This yielded a total of 300 simulations. H-reflex amplitude was expressed as the average percentage of the number of muscle fibers recruited at the appropriate delay (typically $22-27 \mathrm{~ms}$ ) across the five repetitions.

In two additional sets of simulations, the descending drive was set to $2.5 \mathrm{nA}$, while 20 stimuli at $100 \mathrm{~Hz}$ were delivered at $30 \%$ of maximum stimulation intensity $(\sim 10 \%$ below threshold for motor axon recruitment). First, the relation between the MN rheobase current (that spanned a fourfold range across the $\mathrm{MN}$ pool) and the Ia synaptic strength was varied (linearly) so the strength of the Ia projection to the smallest $\mathrm{MN}$ normalized to the MN size (rheobase) was fourfold smaller, equal to, or fourfold larger than that of the largest MN. The total Ia synaptic strength was normalized so it was similar across the three conditions. This implied that the absolute Ia synaptic strength was either relatively constant across all MNs, or skewed (to two different degrees) toward higher strengths for large MNs. In this way, these different settings reflect the contrasting experimental observations across muscles that the Ia fibers may project the strongest on large MNs $(13,61,78,79)$ or on small MNs (7, 76). These across-muscle differences may represent different task-dependent levels of presynaptic inhibition $(3,9,24,74,77,86$, 87 ) affecting the MNs in a size-dependent way $(1,4)$. Next, the 
simulations were repeated with different EPSP duration (EPSC time constant set to $1,1.5$, or 2 ). This range of values reflected not only the inherent variability in the EPSP duration (36), but also the influence on the compound EPSP from other excitatory $(18,50)$ and inhibitory $(17,59,64)$ inputs activated by the peripheral stimulation. As these inputs are primarily polysynaptic (Ia, Ib, and cutaneous) (43) and/or conducted via slower axons (Ib or cutaneous) $(44,45,72)$, they arrive at the MN after the Ia monosynaptic input and can, therefore, be modeled in a simplified way by a shortening (in case of inhibition) or a lengthening (in case of excitation) of the monosynaptic Ia EPSC. The simulations for each of the three settings for distributions of Ia synaptic strength and EPSP duration were repeated 10 times.

Experiments. Seven healthy men $(30.3 \pm 4.8 \mathrm{yr})$ participated in the experiment. The subjects had no known neurological disorders and were not taking any medications at the time of the experiment. Another four subjects were recruited, but were excluded from the study as no measureable H-reflex could be evoked. Ethical approval for the study was granted by the local Ethics Committee, and the procedures were conducted according to the Declaration of Helsinki. Informed consent was obtained from each subject before participation.

The subject was seated in an adjustable chair in front of a table with the proximal part of the left forearm (nondominant for all subjects) resting on a support coated with foam layer (see Fig. 1). The arm was extended in front of the subject with the wrist extended horizontally against gravity and the palm of the hand facing down. A 100-g load was fixed to the hand to induce a low level of voluntary contraction in the wrist extensor muscles equivalent to $\sim 2 \%$ maximum voluntary contraction, assuming a maximum wrist extension force of $50 \mathrm{~N}$ (40). The elbow angle was $\sim 150^{\circ}$.

A disposable stimulation electrode (Krauth+Timmermann, Hamburg, Germany; circular, diameter: $32 \mathrm{~mm}$ ) was placed over the radial nerve distal to the lateral epicondyle. Its exact position was adjusted to evoke a pure wrist extension movement during continuous $100-\mathrm{Hz}$ stimulation. The ground electrode (Krauth+Timmermann; rectangular, $50 \times 90 \mathrm{~mm}$ ) was placed proximal to the elbow. A stimulator (Digitimer, Welwyn Garden City, UK) delivering asymmetrical biphasic pulses was used. Pulse width was set to $400 \mu$ s to optimize recruitment of afferent fibers (83).

Two surface EMG electrodes (diameter 11-mm Ag/AgCl electrodes: Neuroline 720, Ambu) were placed over the extensor carpi radialis longus muscle. The position was verified by palpation of the muscle during wrist extension evoked by continuous $100-\mathrm{Hz}$ stimulation. The skin was carefully cleansed before the electrode placement. At a later stage in the experiment (see Experimental procedure), a pair of Teflon-coated stainless steel wires was inserted via a 25 -gauge disposable hypodermic needle, $\sim 10 \mathrm{~mm}$ proximal to the surface EMG electrodes. The wires were cut to expose only the cross section at the end that was inserted into the muscle. Surface and intramuscular EMG were amplified (EMG2, OTBioelettronica, IT) with bandwidths of $10-500$ and $100-4,400 \mathrm{~Hz}$, respectively, and sampled with a data acquisition card (NI USB 6210, National Instruments) at 2 and $10 \mathrm{kHz}$, respectively.

Experimental procedure. The experiment consisted of three parts within the same experimental session. The first two parts were performed with the subject holding a 100-g load, whereas the load was variable in the third part (see below). In the first part, the H-reflex recruitment curve was obtained by recording the response to stimulations between 4 and $28 \mathrm{~mA}$ (or up to the pain threshold, if lower than $28 \mathrm{~mA}$ ) in steps of $4 \mathrm{~mA}$. The order of the stimulus intensities was randomized. After obtaining the H-reflex recruitment curve, additional current levels were included to achieve a higher resolution of the recruitment curve around its onset and peak. For each intensity, eight stimuli were delivered with an interstimulus interval of $4 \mathrm{~s}$ to minimize the carry-over effect of postactivation depression across trains of pulses (27). The filtered EMG signal was averaged over the eight repetitions, and the H-reflex amplitude was measured as the peak-to-peak amplitude in an interval of 12-22 ms after the stimulus. From the H-reflex recruitment curve, the highest stimulation current at which no H-reflex occurred $\left(I_{\mathrm{thr}}\right)$ and the current required for obtaining an H-reflex with amplitude equal to $50 \%$ of the maximum amplitude $\left(I_{50}\right)$ were identified.

In the second part of the experiment, four current levels evenly spaced in the interval from $I_{\mathrm{thr}}$ to $I_{50}$ were selected, including $I_{\mathrm{thr}}$ and $I_{50}$ as minimum and maximum applied current, respectively. For each of these stimulation intensities, eight repetitions of $1,2,3,4,5,10$, and 20 stimuli were delivered at both 30 and $100 \mathrm{~Hz}$. These two frequencies were selected in order for the interstimulus intervals to be longer and shorter of the presumed EPSP duration of $\sim 15 \mathrm{~ms}$, respectively. Between the bursts of stimulation, there was a pause of $4 \mathrm{~s}$, and the order of the repetitions of each number of stimuli was randomized. In this way, eight trials (four stimulation intensities $\times$ two stimulation frequencies) of 56 sets (eight repetitions of the seven numbers of stimuli within each set) were delivered. The order of these trials was also randomized. Three minutes of rest were given between each trial to minimize fatigue and discomfort. In a similar way as for the H-reflex recruitment curve (part 1), the average of the eight EMG recordings for each condition (number of stimuli, intensity, and frequency) was used to estimate the amplitude of the neural responses (see Data processing and statistical analysis).

Finally, in the third part of the experiment, the intramuscular electrodes were inserted proximal to the surface electrodes, as explained before. For the intramuscular recordings, the load added to the hand was progressively increased from the initial $100 \mathrm{~g}$, in steps of $100 \mathrm{~g}$, until regular discharges of a clear single motor unit (target unit) could be identified from the intramuscular signals. After identifying the load for the recruitment of the target motor unit, the load was reduced to the level immediately before the recruitment ( $100 \mathrm{~g}$ less) so that the force exerted was just below the recruitment threshold of the target unit. The additional applied weights were in the range $0-600 \mathrm{~g}$ $(<200 \mathrm{~g}$ for $5 / 6$ subjects) across subjects, implying that target units

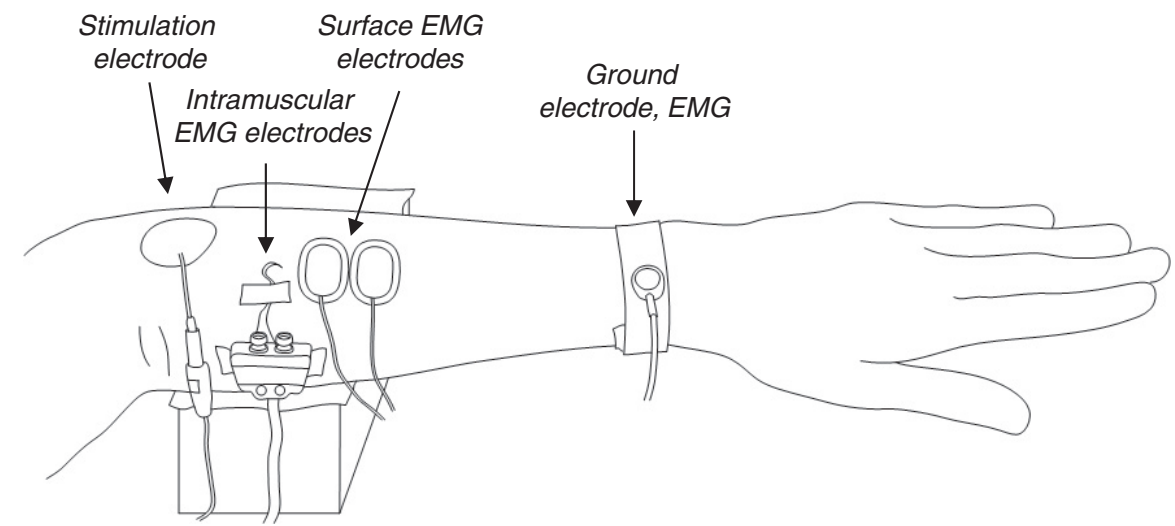

Fig. 1. Experimental setup. Stimulation electrode location varied across subjects according to where a pure extension was evoked. Ground electrode for stimulation (not shown) was located proximal to the elbow. Intramuscular electrodes were inserted 5-10 $\mathrm{mm}$ proximal to the surface electrodes. The proximal part of the lower arm rested on a box with foam padding on top. 
A

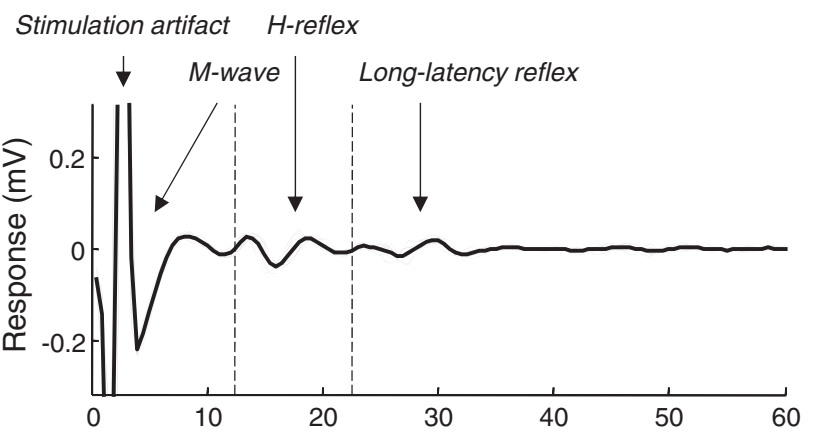

B

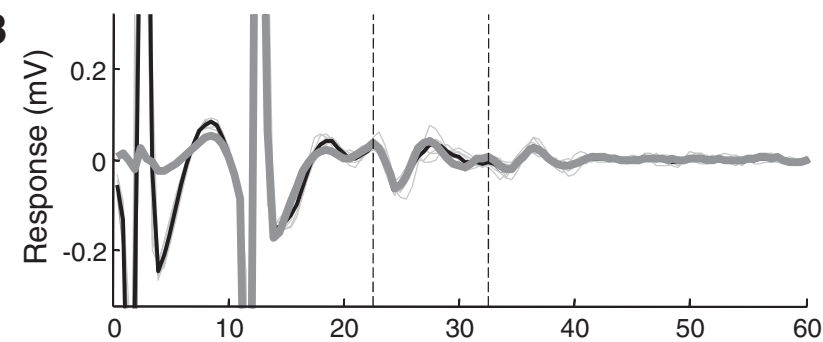

C

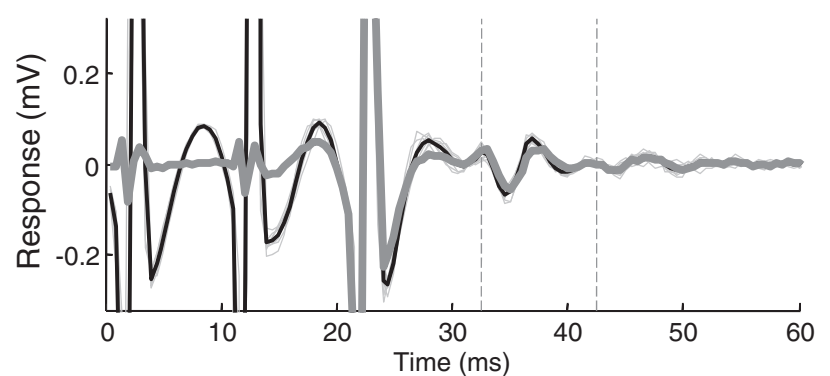

Fig. 2. A-C: the surface EMG recordings during one to three stimuli, respectively, delivered at $100 \mathrm{~Hz}$ from one representative subject. Thin, light shaded lines indicate the eight individual recordings, and the solid line indicates the average response, from which the reflex amplitudes were estimated. The stimulation intensity was $9 \mathrm{~mA}$, enough to elicit an H-reflex of $\sim 50 \%$ of the maximum amplitude with one stimulus. In $A$, the different components of the signal are indicated. The stimulation artifact is a high-amplitude triphasic burst, almost completely overlapping with the M-wave due to the very short distance between the stimulation electrode and the EMG electrodes. The H-reflex occurs after 12-22 ms. The vertical dashed lines indicate the period in which the H-reflex was identified across conditions with different number of stimuli. Finally, the long-latency reflex occurs with a delay of $\sim 30$ ms with respect to the stimuli. The thick, dark shaded lines in $B$ and $C$ represent the estimated response purely to the second and third stimulus. The pure response to the second stimulus was obtained by subtracting the response to the first stimulus (solid line in $A$ ) from the compound response to the second stimulus (solid line in $B$ ), and similarly for the following number of stimuli. In this way, the influence of any overlap of, e.g., the long-latency reflex, from the first stimulus on the H-reflex of the second stimulus was eliminated, and the corrected H-reflex amplitude was used for further analysis.

were low-threshold motor units, as these contraction levels were assumed to be $<10 \%$ maximum voluntary contraction (40). Next, the multistimuli protocol described above was repeated, but this time with eight repetitions of one to six stimuli at $100 \mathrm{~Hz}$. Four trials with different stimulation intensities were applied: $I_{\mathrm{thr}}$, as determined in the part 1 , and 1,2 , and $3 \mathrm{~mA}$ above $I_{\text {thr }}$. The order of these trials was randomized, with a rest of $3 \mathrm{~min}$ between trials. Between any two trials, the recruitment of the target unit was confirmed by adding $100-\mathrm{g}$ load for a brief interval of time.

Data processing and statistical analysis. The surface EMG signals were offline high-pass filtered (Butterworth, zero-phase, 5th order) with a cut-off frequency of $120 \mathrm{~Hz}$ to minimize the risk of overlap between the H-reflex and the stimulation artefact and/or the M-wave. The intramuscular EMG signals were offline high-pass filtered (Butterworth, zero-phase, 4th order) with a cut-off frequency of $1,000 \mathrm{~Hz}$ for better discrimination of individual motor units.

In most subjects, a small long-latency reflex response occurred in the range of 10-15 ms after the H-reflex (Fig. 2). This latency implied that this reflex component from one stimulus could overlap with the $\mathrm{H}$-reflex response of the following stimulus when delivering multiple stimuli at $100 \mathrm{~Hz}$ (part 2). Therefore, the long-latency reflex in the trial with $N-1$ stimuli was used as a template and was subtracted from the recorded responses to $N$ stimuli. Figure 2 illustrates this approach. This subtraction could not be performed for 10 and 20 stimuli since trains consisting of exactly 9 and 19 stimuli were not included in the measures. In these cases, the template to subtract was obtained from the time-shifted long-latency response from the fifth stimulus. The H-reflex amplitudes were estimated as the peak-to-peak amplitude of the corrected response in the 12- to 22-ms range after the stimulus and normalized to the maximum H-reflex amplitude identified in part 1 of the experiment. Next, the normalized H-reflexes for the cases with multiple stimuli $(2-5,10,20)$ were expressed as the difference from the single-stimulus case. Linear regression was applied to investigate the relation between this normalized H-reflex increment related to each of the seven different number of stimuli and the H-reflex amplitude obtained with one stimulus. In this way, both variables were expressed in units of normalized H-reflex amplitude. Regression significance was tested using $F$-test, and the slopes of these linear fits were compared across conditions using the Student's $t$-test. ANOVA analysis was applied to investigate the long-latency reflex amplitude and its determinants.

From the four trials of intramuscular EMG per subject (part 3), we identified the stimulation intensity immediately below the intensity at which the target motor unit was recruited on a regular basis. Target motor unit recruitment was identified by applying a threshold above the baseline noise level (at least 2 SDs) to the EMG recording in a window of 12-22 ms after the stimulus. The trials with EMG activity above this threshold were aligned according to their peak values and averaged. Only if the shapes of the action potentials were consistent

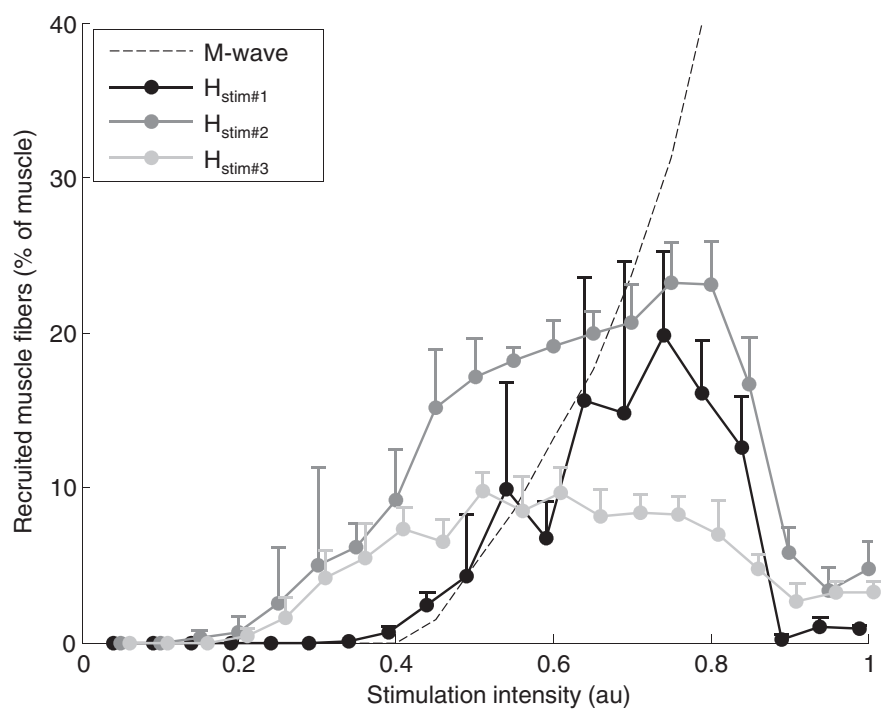

Fig. 3. The simulated H-reflex recruitment curves (muscle activation vs. stimulation intensity) for one, two, and three stimuli at $100 \mathrm{~Hz}$ (black, dark shaded, and light shaded lines, respectively) along with the M-wave amplitude (dashed line). Muscle excitation level was approximately one-half of that required for recruitment of the first motor unit. Stimulation intensity is expressed in arbitrary units as the range of intensities required for activation of the first axon (0) to the intensity required for activation of all axons (1). 
(by visual inspection), the motor unit was included for further analysis. In this way, we analyzed the intramuscular signals obtained at this stimulation level and at the level of $1 \mathrm{~mA}$ above it by counting the number of instances the target motor unit elicited action potentials for each number of stimuli. Two subjects were excluded from this part of the analysis. One presented an interference intramuscular EMG with no clear distinction of individual motor units, even at the lowest load, and, for the other, the target motor unit could not be reliably identified across all trials.

\section{RESULTS}

Simulations. Figure 3 shows the simulated H-reflex recruitment curves as obtained for different numbers of stimuli at 100 Hz. Due to the relatively low background excitation level, a stimulation intensity equivalent to the activation of $35 \%$ of the axons was required for triggering a $\mathrm{MN}$ action potential with only one stimulus (first data point $>0$ for the black line; Fig. $3)$. At this intensity, 15 to 25 out of the 54 Ia axons were recruited. The recruitment curves for the responses to two and three stimuli, however, showed MN recruitment thresholds at much lower intensities, with substantial muscle activity at 35\% stimulation intensity. Thus, even though the first stimulus was not sufficient for the MN membrane potentials to reach their thresholds, it served to depolarize the MNs so that there was a greater probability that one of the next EPSCs would trigger an action potential, as hypothesized. At higher stimulation intensities $(>60 \%)$, three stimuli activated fewer muscle fibers than in the single-stimulus case. This depression reflected the fact that, at the third stimulus, the majority of the MNs were in
A

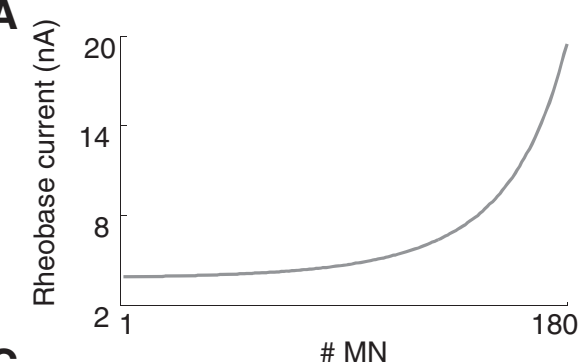

C

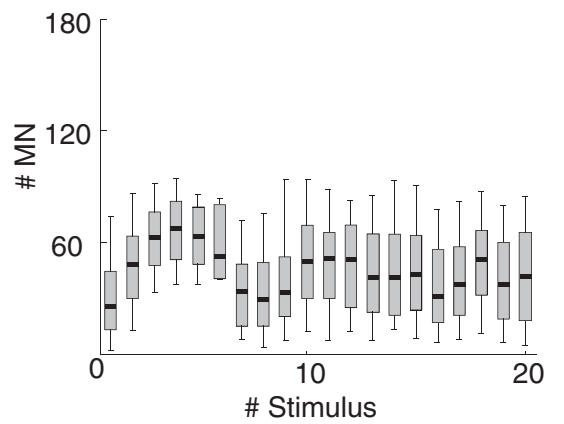

$\mathbf{F}$
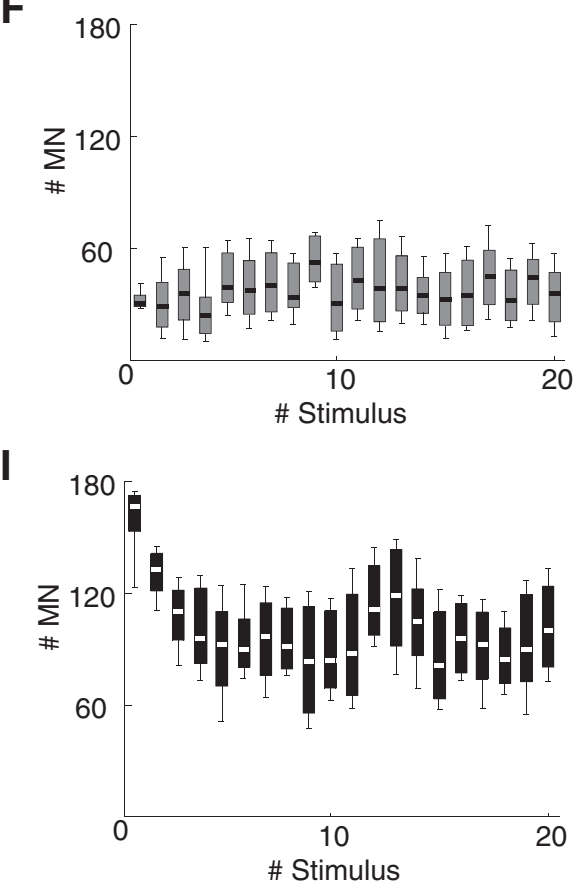

B

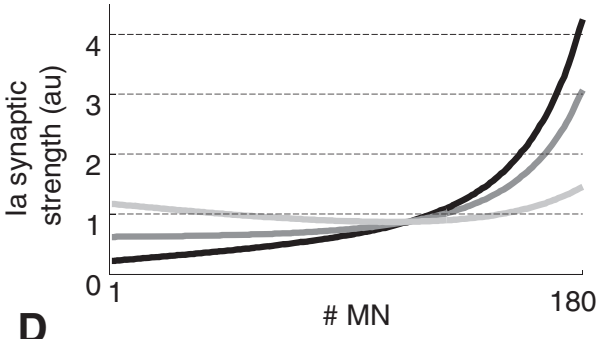

D
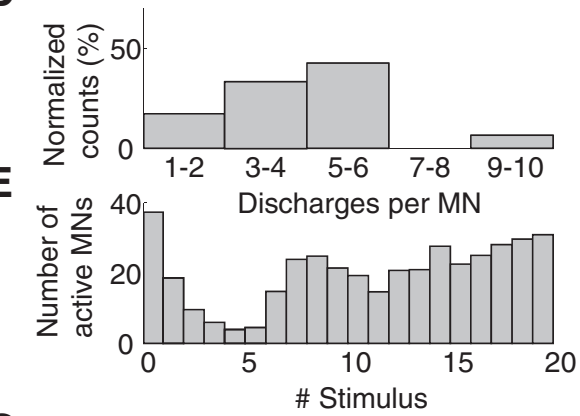

G

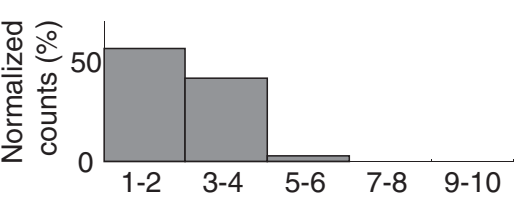

H

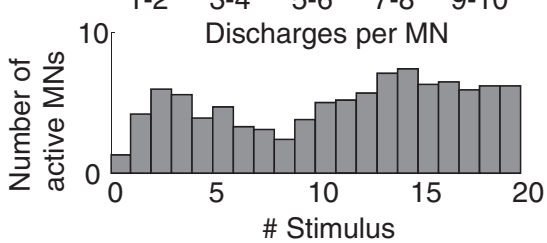

J

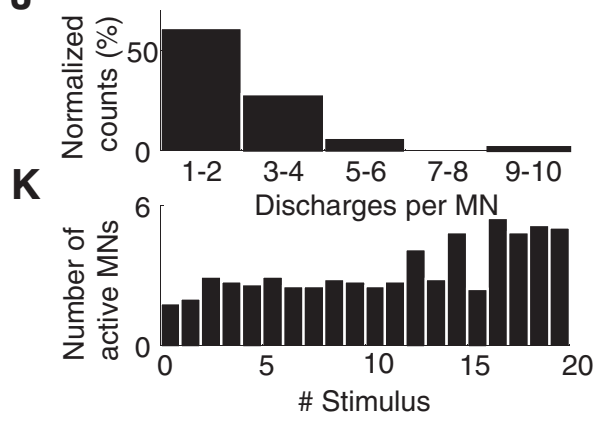

Fig. 4. The simulated rheobase current for all 180 motoneurons (MNs; $A$ ) and the three simulated distributions of normalized Ia synaptic strengths across the MN pool (B). In $B$, the light shaded line reflects the simulations in which the Ia strength for each MN normalized to its rheobase current was fourfold higher for the smallest MN (no. 1) compared with the largest one (no. 180), the dark shaded line reflects the simulations with equal normalized Ia strength across the MN pool, and the solid line, the simulations with normalized Ia strength fourfold lower for the smallest MN compared with the largest. This color coding is used in the lower panels, so $C, D$, and $E$ reflect the results from the first simulation condition; $F, G$, and $H$ for the second; and $I$, $J$, and $K$ for the last condition. $C, F$, and $I$ depict the range of MNs that were recruited by the stimulation. $D, G$, and $J$ show the distribution of discharges per each MN during the 200-ms stimulation duration. In $D, \sim 40 \%$ of all active MNs elicited five to six action potentials [equivalent to a discharge rate of 25-30 pulses/s (pps)], whereas $>50 \%$ of the recruited MNs elicited one to two action potentials ( $<10 \mathrm{pps})$ in the condition of $J . E, H$, and $K$ depict the number of active MNs per each of the 20 stimuli. 
hyperpolarized states after having discharged action potentials at either one or two stimuli. In all cases, the H-reflex recruitment curves were depressed at high stimulation intensities $(>80 \%)$ due to antidromic collisions in the motor axons.

Figure 4 illustrates the influence of the three different distributions of Ia synaptic strength across the MN pool (Fig. 4B) on the recruitment during 20 submotor threshold stimuli delivered at $100 \mathrm{~Hz}$. When the synaptic strength relative to the rheobase current was fourfold higher for the smallest MN compared with the largest one, low-threshold MNs (less than MN no. 90) were recruited (Fig. 4C) with an average number of recruited MNs per stimulus of 20.2. The majority of the recruited MNs elicited 3-6 action potentials in response to the 20 stimuli, equivalent to discharge rates in the range 15-35 pulses/s (pps) (Fig. 4D). These discharges were evenly distributed throughout the simulation period. In comparison, in the simulations where the synaptic strength was proportional to the rheobase current, fewer MNs in the same range (low-threshold) were recruited (5.0 MNs per stimulus, primarily at discharge rates $<20$ pps; Fig. $4, F, G$, and $H$ ). Similarly, when the Ia strength relative to the rheobase current was fourfold higher in the largest MN, few MNs were recruited at low rates (1.4 MNs per stimulus, primarily at discharge rates $<10 \mathrm{pps}$; Fig. $4, J$ and $K$ ), but at this setting primarily high-threshold MNs were active (Fig. 4I). These differences imply that MNs for which the relative EPSP is the highest are most likely to be recruited by the stimulation. However, less current (and thus less temporal summation) is required to bring the membrane potential of the small MNs to the threshold for generation of action potentials, and, since they are usually most prevalent in the MN pool (34), these MNs will, when recruited, tend to be active in higher numbers and at higher discharge rates. An interesting observation was that the number of active MNs per stimuli tended to oscillate for the first $\sim 10$ stimuli before reaching a stable level (Fig. 4, $E$ and $H$ ). In the conditions shown in Fig. $4 H$, this reflected that most MNs capable of being activated by the stimulation needed the summed input from two to four stimuli to generate action potentials, after which this group of
MNs was hyperpolarized for the duration of the following stimuli. Synaptic noise and small differences in the intrinsic properties, however, implied a certain variability in the number of additional stimuli required for another action potential, which meant that the number of active MNs eventually reached a stable level.

The influence of the EPSP duration on the MN recruitment during $100-\mathrm{Hz}$ stimulation is depicted in Fig. 5. Short and long durations reflected high levels of either inhibition or excitation arriving at the MN after the Ia monosynaptic volley, respectively. When the EPSP was short, progressively fewer MNs were recruited per stimulus (Fig. 5B), and the recruited MNs got less and less active, with $66 \%$ of the active MNs exhibiting discharges rates $<10$ pps (Fig. $5 C$ ).

Experiments. A long-latency reflex occurred, on average, $12.7 \pm 2.7 \mathrm{~ms}$ after the H-reflex. Across all subjects, number of stimuli, and both frequencies, its amplitude was $6.9 \pm 1.9$, $7.6 \pm 2.0,12.6 \pm 5.0$, and $14.7 \pm 5.5 \%$ of the maximum H-reflex amplitude, for the lowest to highest intensities, respectively. Previous studies of the extensor carpi radialis longus muscle have reported additional reflex responses with latencies of up to $8 \mathrm{~ms}$ (presumably due to polysynaptic excitation) (58) and, on average, $25 \mathrm{~ms}$ (presumably related to movement in the unconstrained hand) (82) with respect to the H-reflex. Four-way ANOVA analysis revealed a significant influence of subject and stimulation intensity on the longlatency reflex $(P<0.0001$ for both variables $)$, but not for number of stimuli $(P=0.43)$ and frequency $(P=0.20)$. As the number of stimuli did not influence the amplitude, we assumed that using the time-shifted response of the 5th stimulus as a template for the responses to the 9th and 19th stimuli to estimate the pure responses to the 10th and 20th stimuli did not introduce a bias in the results.

Figure 6 depicts the H-reflex recruitment curve for one stimulus in one subject. As typical, the H-reflex amplitude increased with the stimulus intensity until it reached a maximum value after which the amplitude decreased. For this subject, currents above $18 \mathrm{~mA}$ were not applied due to dis-
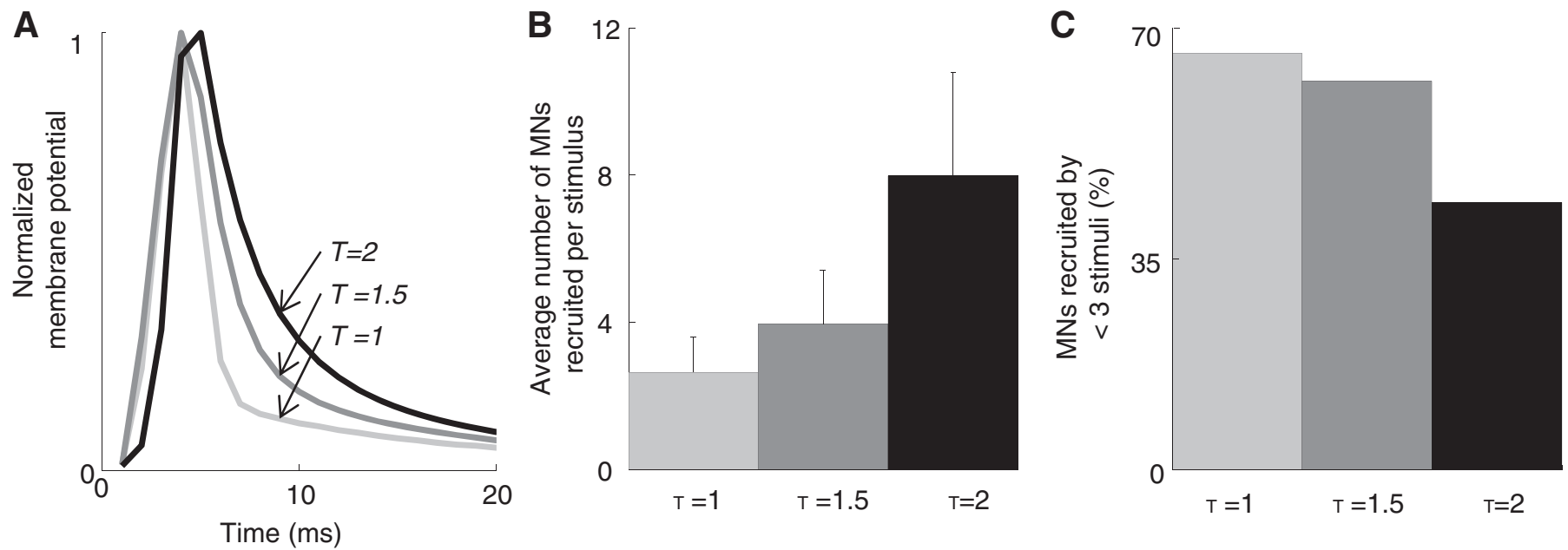

Fig. 5. A: the average Ia excitatory postsynaptic potentials (EPSPs) for the three different time constants of the Ia excitatory postsynaptic current (EPSC) (solid: $\tau=2$; dark shaded: $\tau=1.5$; light shaded: $\tau=1$ ) simulated in the absence of any other MN inputs. $B$ and $C$ depict the effect of the different EPSP durations on the MN recruitment during 100-Hz stimulation for $200 \mathrm{~ms}$, as the average number of MNs recruited per stimulus $(B)$ and the proportion of recruited MNs eliciting one to two action potentials (equivalent to a discharge rate $<10 \mathrm{pps} ; C$ ). Color coding in $B$ and $C$ reflects the different EPSC time constants shown in $A$. 


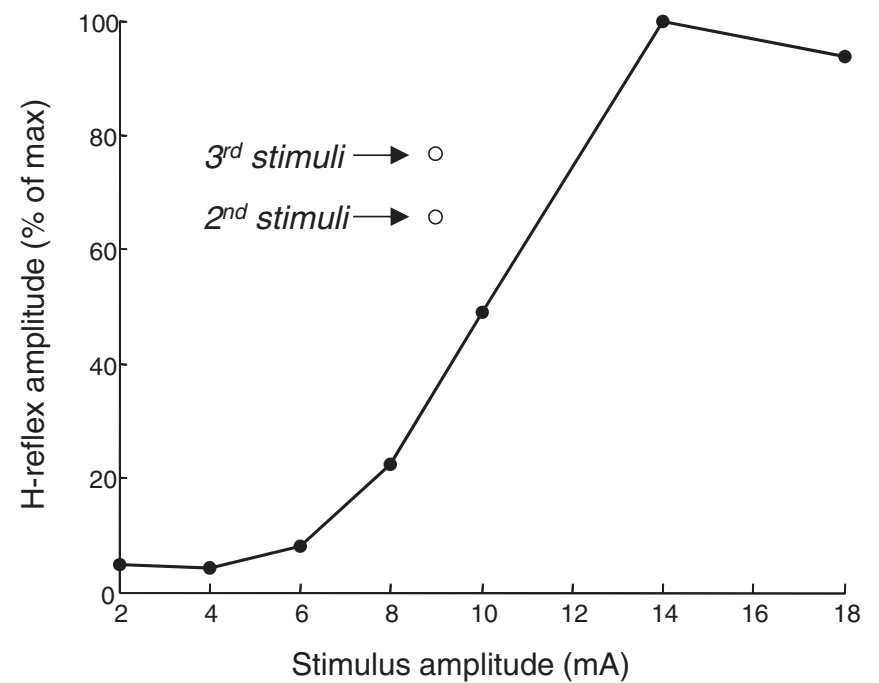

Fig. 6. The H-reflex amplitude as a function of the stimulation intensity for one representative subject when only one stimulus was delivered (solid connected circles). The open circles indicate the H-reflex amplitude in response to two or three stimuli with an amplitude of $9 \mathrm{~mA}$ at $100 \mathrm{~Hz}$. The amplitude of the H-reflex increased substantially when more than one stimulus was delivered.

comfort. The H-reflex amplitudes in response to two and three stimuli at $100 \mathrm{~Hz}$ are shown at the intensity of $9 \mathrm{~mA}$, which corresponds to the highest of the four current levels between $I_{\text {thr }}$ and $I_{50}$. In these cases, the H-reflex amplitude was substantially greater than for the recruitment curve with a single stimulus. For example, the H-reflex amplitude obtained with three pulses at $9 \mathrm{~mA}$ was more than twice the amplitude for a single stimulus, reaching almost $80 \%$ of the maximum single pulse H-reflex amplitude $(0.14 \mathrm{mV}$ at $14 \mathrm{~mA})$. This observation was generally valid across subjects, as illustrated in Fig. 7 . Here, the H-reflex amplitudes for all number of stimuli across four stimulation intensities are shown for 30 and $100 \mathrm{~Hz}$. At 30 $\mathrm{Hz}$, the H-reflex amplitude often decreased when more than one stimulus was delivered. For example, the H-reflex amplitude at $9 \mathrm{~mA}$ for subject 1 (Fig. 7A) decreased slightly for two to five stimuli, and more dramatically for 10 and 20 , whereas the response exhibited a more rapid decrease with respect to the single-stimulus case in other subjects (Fig. 7, $C$ and $E$ ). On the contrary, at $100 \mathrm{~Hz}$, the response tended to increase substantially when more than one stimulus was used (e.g., Fig. $7 B$ ). The peak responses were usually obtained in response to two to four stimuli, while a depression with respect to the first stimulus was observed for higher numbers of stimuli in some subjects.

The average magnitudes of the increment in the H-reflex amplitude relative to multiple stimuli at the two frequencies are depicted in Fig. 8. In this figure, the stimulation intensities are expressed not as a current, but by the H-reflex amplitude evoked by that current expressed as a percentage of the maximum single-pulse H-reflex amplitude. The $y$-axis represents the increment for a given number of stimuli $(3,5$, and 20 stimuli are shown) with respect to the single-stimulus case, also normalized to the maximum single pulse H-reflex. The results for 2, 4, and 10 stimuli were comparable to those shown in Fig. 8. In this way, the results from all subjects could be directly compared and evaluated using linear regression. As indicated by the figure, $100-\mathrm{Hz}$ stimulation generally involved a larger increment in the H-reflex amplitude compared with 30-Hz stimulation. In several cases (e.g., Fig. 8, $C$ and $E$ ), $30-\mathrm{Hz}$ stimulation involved a depression of the H-reflex, as indicated by the negative slope, whereas $100 \mathrm{~Hz}$ always led to an increase (e.g., Fig. 8, $B$ and $D$ ). For $100-\mathrm{Hz}$ stimulation, the positive correlation was significant in the cases for $2-4$ stimuli $(P=0.0003, P=0.001, P=0.0014$, respectively $)$, whereas there was a significant negative correlation at 10 stimuli for $30-\mathrm{Hz}$ stimulation $(P=0.001)$. At a stimulation intensity required for $50 \%$ of the maximum H-reflex, the slope coefficients of the linear regression predicted that five stimuli involved an average depression of the H-reflex amplitude of $10 \%$ at $30 \mathrm{~Hz}$, but an average increase of $8 \%$ at $100 \mathrm{~Hz}$. On average, the slopes of the regression lines for all number of stimuli were $-0.08 \pm 0.18$ for $30 \mathrm{~Hz}$ and $0.25 \pm 0.16$ for $100 \mathrm{~Hz}$. The average difference in the slopes across the two frequencies for
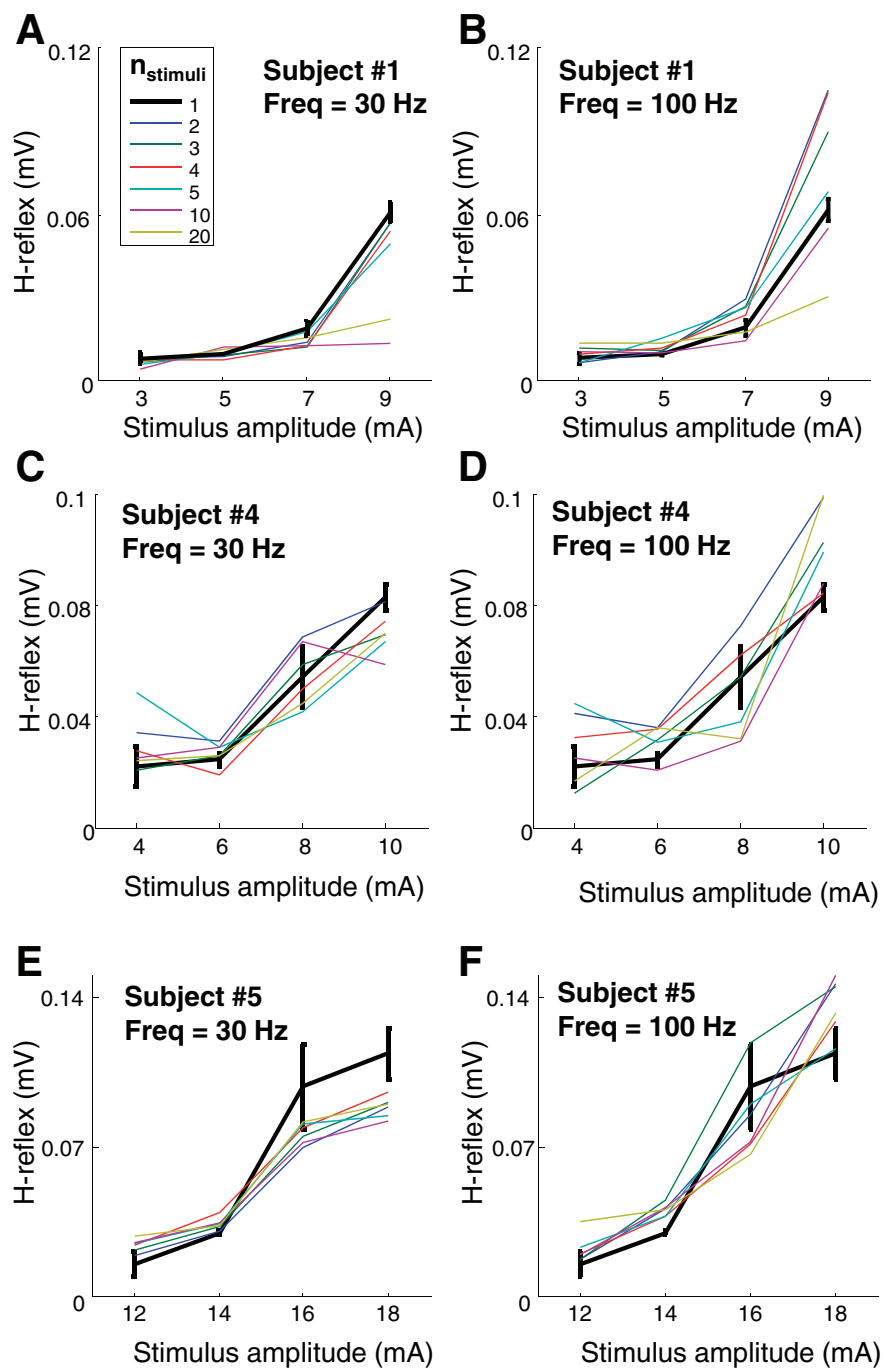

Fig. 7. The H-reflex amplitude in response to $1-5,10$, or 20 stimuli delivered (see color coding in inset in $A$ ) at four different stimulation intensities (approximately equivalent to $0-50 \%$ of the intensity required for maximum single-stimulus H-reflex amplitude) at $30 \mathrm{~Hz}(A, C$, and $E)$ and $100 \mathrm{~Hz}(B, D$, and $F$ ) for three representative subjects [subject 1 ( $A$ and $B)$, subject 4 ( $C$ and $D)$, subject $5(D$ and $F)$ ]. As the condition indicated by the black line (one stimulus) is similar across the two frequencies, the average and SD of these two measurements are shown. 
A

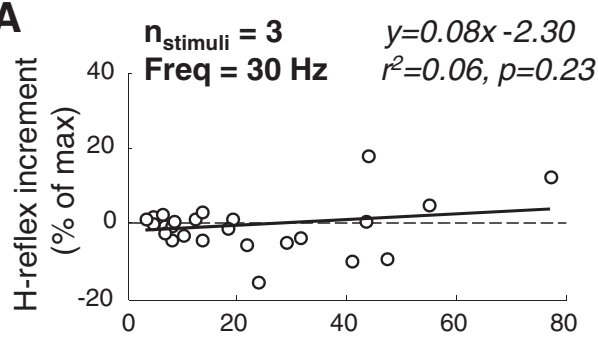

Fig. 8. The change in H-reflex amplitude when multiple stimuli are delivered at $30 \mathrm{~Hz}(A, C$, and $E)$ and $100 \mathrm{~Hz}(B, D$, and $F)$ with respect to the amplitude achieved with only one stimulus for all subjects. Each circle represents one stimulation intensity for one subject. Stimulation intensity $(x$ axis) is represented as the amplitude of the equivalent H-reflex obtained with one stimulus. Here, the results from 3 ( $A$ and $B), 5(C$ and $D$ ), and 20 stimuli $(E$ and $F)$ are shown. The solid line indicates the best linear fit to the data, whereas the dashed line represents zero H-reflex increment.

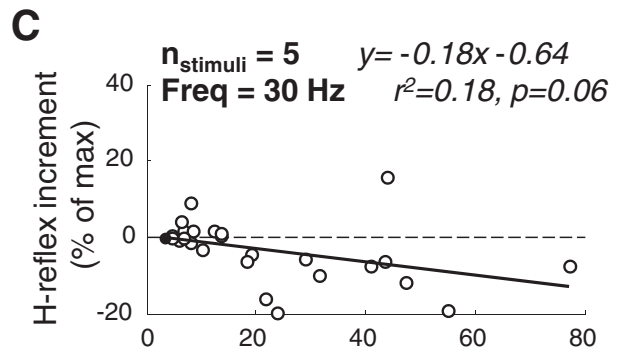

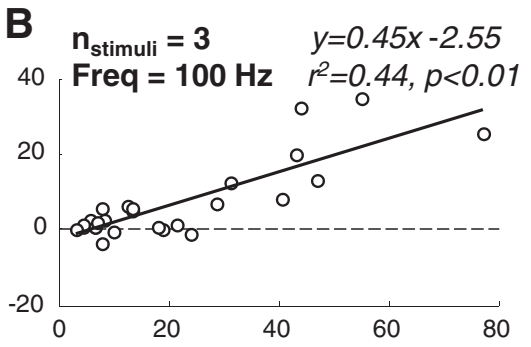

E

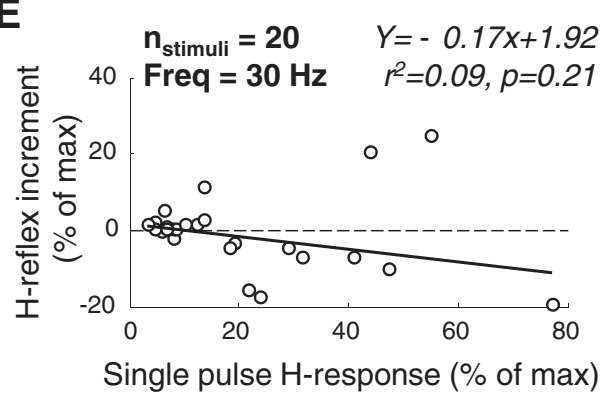

D

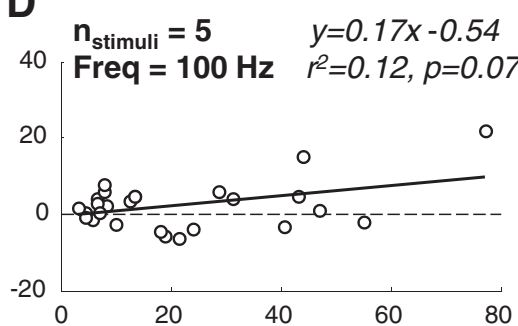

$\mathbf{F}$

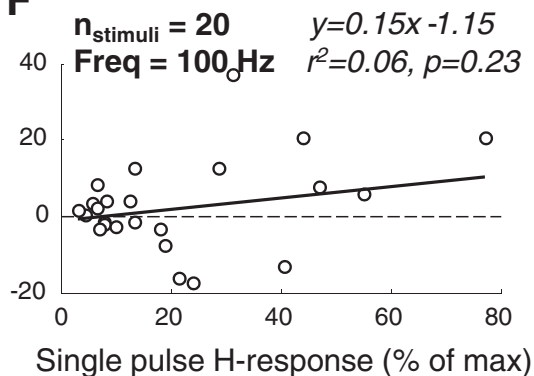

each number of stimuli was $0.32 \pm 0.07$ (statistically different from $0 ; P<0.0001$ ).

Figure 9 shows the results from the intramuscular EMG data from the five subjects in which the target motor unit was reliably tracked throughout the stimulation protocol. Each row of panels represents one subject. The right column shows the shapes of the motor unit action potential of the target units (aligned and averaged). In most cases, the averaged shape of the action potential (black line) was very similar to the single traces (shaded lines), indicating a high certainty that all recorded action potentials belonged to the same motor unit. In one case (subject 3; Fig. 9D), a high level of background noise appeared to compromise the similarity of the individual action potentials, whereas in another case superimpositions from another motor unit action potential were sometimes present (subject 6; Fig. 9H). The left column illustrates the number of action potentials for each of the eight repetitions of each number of stimuli (stimuli 1-6). In this way, the histograms represent the probability of the target motor unit recruitment, depending on the input parameters (number of stimuli and stimulation intensity). At the highest stimulation intensity (shaded bars), there was a specific number of stimuli in each subject for which the target unit exhibited a higher probability of eliciting an action potential. This critical number varied across subjects, but was typically between three and five stimuli. This indicates that the neural input from the EPSC from a single pulse of the stimulated afferent fibers was too low to elicit an action potential in this $\mathrm{MN}$, but when multiple pulses arrived in short intervals, the summed input increased the probability of bringing the $\mathrm{MN}$ membrane potential to its threshold. Interestingly, if the MN had a higher tendency of being active after a relatively low number of pulses, the likelihood of a discharge at higher number of stimuli decreased, indicating hyperpolarization (relative refractory period) of the MN membrane potential at the times of arrival of the following stimuli. Finally, for one subject (subject 7; Fig. $9 I$ ), the target unit was recruited at the low stimulation intensity (7 mA) by four to five stimuli. However, when the stimulation intensity (and thereby the net input to the $\mathrm{MN}$ ) was increased by $1 \mathrm{~mA}$, only three to four stimuli were needed. Again, this indicates that temporal summation over multiple stimuli is required for action potentials to occur when stimulating at low intensities.

\section{DISCUSSION}

This study investigated the output of the pool of MNs innervating the extensor carpi radialis longus muscle during low-level, high-frequency nerve stimulation. We found that multiple pulses delivered at $100 \mathrm{~Hz}$ substantially increased the amplitude of the H-reflex with respect to single pulses. This increase was persistent for up to 20 pulses, but was highest for the first 2 to 4 . The same summation effect was not observed during $30-\mathrm{Hz}$ stimulation. Furthermore, using recordings of single motor unit activity, it was observed that, during highfrequency stimulation, many MNs tended to fire only after a 
A

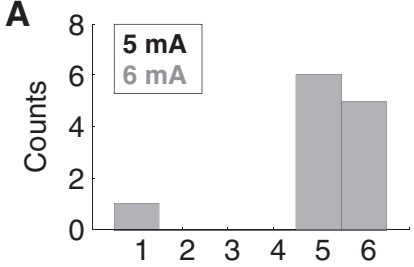

C

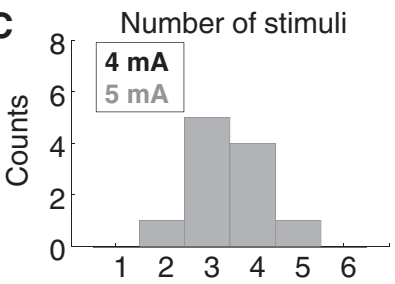

E

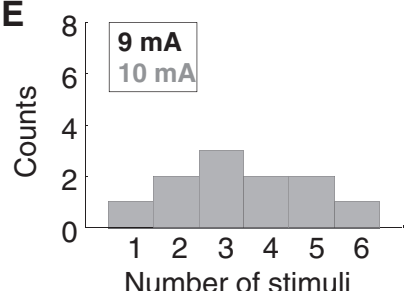

G

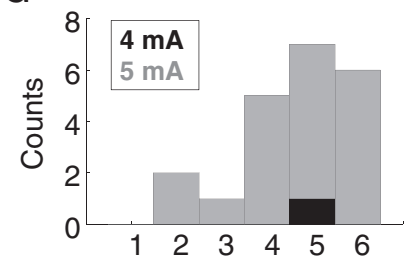

I Number of stimuli

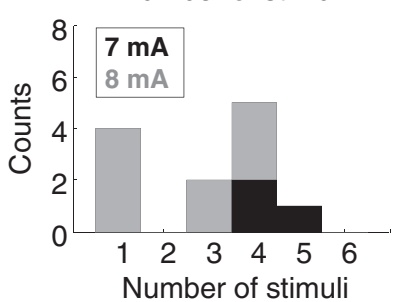

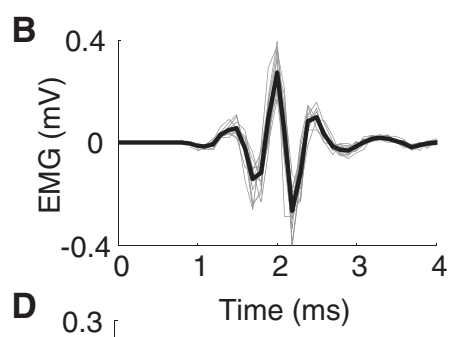
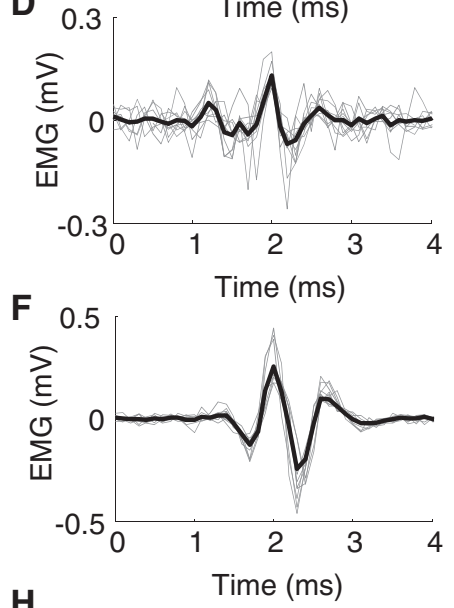

H
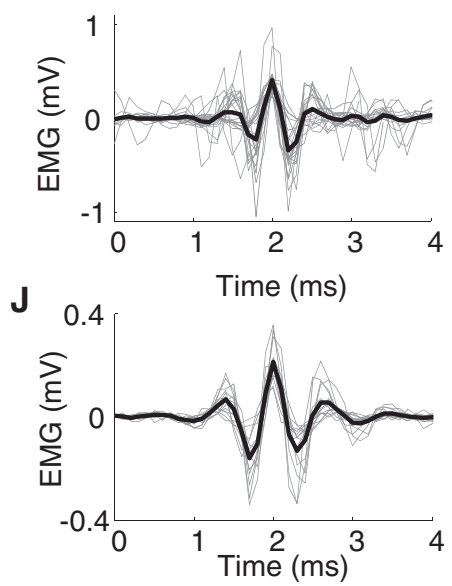

Fig. 9. The occurrences of action potentials of the target motor unit in response to the eight repetitions of one to six stimuli at $100 \mathrm{~Hz}$. A, C, E, G, and $I$ show histograms quantifying the number of action potentials to each number of stimuli for subject 1,3,5,6, and 7, respectively. Solid bars indicate the counts for the lower (subthreshold) stimulation intensity, whereas the shaded bars indicate the counts for a stimulation intensity $1 \mathrm{~mA}$ above this level. $B, D, F$, $H$, and $J$ show the shapes of the action potentials of the target motor units (corresponding to the histogram to the left of it). Shaded lines represent the single action potentials, and the thick solid line, the average action potential.

certain number of stimuli. The number of needed stimuli depends on intrinsic MN properties, background excitation, and magnitude of the input. Since a different subset of MNs responds to each stimulus pulse, continuous activation of the muscle could be achieved with individual $\mathrm{MN}$ discharge rates severalfold lower than the stimulation rate (unlike traditional NMES). This observation was reproduced by the realistic computer simulations showing a stable number of MNs being activated per stimuli with discharge rates within physiological ranges (34). In most simulation settings, the MNs were recruited in a size-ordered fashion. Inverse recruitment was present only when the Ia fibers projected very strongly to

high-threshold MNs (Fig. 4I). Experimentally, such reflex recruitment has been observed for the masseter muscle (78), whereas other muscles exhibit mixed recruitment biased toward either large (79) or (as in the case of extensor carpi radialis longus) small (76) MNs. The simulations were conducted at a fixed subthreshold background excitation level, but increasing the MN pool excitability would increase the number of recruited MNs in a predictable way: in the cases where small MNs were recruited first, an increased background excitation would recruit progressively larger MNs, while the smaller MNs would be activated by fewer stimuli. Furthermore, modulations in the EPSP amplitude, for example, due to stimulation location (10), task dependency (30), fatigue (31), or training state (81), would change the activation of the MN pool. A similar effect was achieved by changing the EPSP duration, but the simulations showed that $\mathrm{MN}$ recruitment has a considerable safety margin with respect to such changes that may occur due to, e.g., polysynaptic Ib inhibitory input (45) or time-dependent changes in MN excitability due to activation of cutaneous afferents (38). While the simulations showed that multiple stimuli can elicit $\mathrm{H}$-reflexes at currents where one stimulus was insufficient (Fig. 3), this was only observed experimentally in few subjects (Fig. 7) and not in a consistent way (Fig. 8). This discrepancy may be explained by the fact that a higher background excitation was present in the experiment (due to the low-level contraction), making an H-reflex likely to occur even when only few afferent fibers were activated. Furthermore, it is possible that a relatively large number of Ia axons with similar, low rheobase currents were recruited between two steps of current. This may likely have happened due to the 2-mA step-size between stimulation intensities. In this case, a single EPSP at the lowest current involving any Ia axon activation may have been high enough to elicit an H-reflex. Finally, the simulations may have overestimated the variability in Ia conduction velocity (and thus rheobase current), as a lower and more compressed distribution of conduction velocities has been reported for some human muscles $(7,17)$.

Several phenomena may explain the elevated H-reflex amplitudes at $100-\mathrm{Hz}$ stimulation. Posttetanic potentiation can increase $\mathrm{H}$-reflex responses, but this typically requires stimulation at frequencies $>200 \mathrm{~Hz}$ in periods from $10 \mathrm{~s}$ to several minutes $(15,27,33,48)$. This suggests that this was not the primary mechanism underlying the $\mathrm{H}$-reflex elevation observed in the present study, as it occurred after only two stimuli (10 ms). Alternatively, activation of PICs by high-frequency stimulation may have increased the MN excitability, thereby increasing the reflex response amplitudes. Accordingly, it is presumed that such currents can be activated at $100-\mathrm{Hz}$ stimulation (and not at $\sim 30 \mathrm{~Hz}$ ) $(29,49)$ and at the similar pulse widths as that applied in this study (52). However, this has only been demonstrated after seconds of stimulation $(10,23)$ and increased asynchronous activity (10) or self-sustained firing (22), which are observations often related to PIC activation that were not observed in the present study. Finally, a frequencydependent difference in presynaptic inhibition and decreased sensory nerve rheobase current at high-frequency stimulation (47) may, in part, explain the results. Although none of these explanations can be discarded, we believe that the most likely explanation for the elevated $\mathrm{H}$-reflex amplitude at $100-\mathrm{Hz}$ stimulation is the temporal summation of the Ia EPSP in the MNs, as the duration of the compound EPSP $[\sim 15 \mathrm{~ms}(71,80)]$ 
implies that an overlap of subsequent evoked potentials occurred. Additionally, it should be noted that the simulations conceptually replicated the experimental findings (Figs. 3 and 4) without including the changes in synaptic strength or MN intrinsic properties (e.g., EPSP amplitude, PIC). Temporal summation as the underlying mechanism is also compatible with the lack of an increase in the H-reflex amplitude at higher interstimulus intervals (30-Hz stimulation; Fig. 8). The observed decrease in reflex amplitude at this stimulation frequency is in line with previous observations that the H-reflex decreases in response to repetitive stimulation at similar frequencies $(20,25)$. This postactivation depression is presumably due to partial depletion of neurotransmitter at the synapse. The increased H-reflexes at high-frequency stimulation, however, indicated that temporal summation occurring at $100-\mathrm{Hz}$ stimulation is sufficiently strong to overcome this decrease in synaptic strength, but may also reflect the fact that the degree of postactivation depression is relatively low at high frequencies $(25,27)$. When more than five stimuli were applied at 100 $\mathrm{Hz}$, the elevated $\mathrm{H}$-reflex amplitudes observed during the first few stimulus pulses declined. This may be due to a progressive increase in the magnitude of the depression after multiple high-frequency pulses, as previously reported (27), but may also reflect the natural activity cycle of the active MNs demonstrated in the simulations (Fig. 4H). On average, however, an increase in the H-reflex amplitude in response to the 20th stimulus with respect to the 1 st stimulus was observed. It should be noted that a large cross-subject variability in the effect of high-frequency stimulation was observed, in particular in the response to 10 and 20 pulses (Fig. 8). Cross-subject variability in the magnitude at which the postactivation depression occurred and in the amplitude and time constant of the EPSC may partly explain this variability. Furthermore, the sensitivity of the H-reflex amplitude to arm position (30) and mental state of the subject (39) may have contributed as well. Variability due to these factors inevitably occurred to some degree due to the relatively long duration of the experiment $(2-3 \mathrm{~h})$.

The latencies of the H-reflex are not often systematically reported. However, in the present study, the observed latencies (12-20 ms) were shorter than most, but not all, other reports for the same muscle. Typical values in healthy young adults are $18-30 \mathrm{~ms}(28,66,68,84)$, but average latencies of $14-16 \mathrm{~ms}$ with considerable standard deviations have been reported $(9$, 82). Several factors may have contributed to this discrepancy. For example, since the muscle fiber conduction velocities are $\sim 10$ to 20 times smaller [normally $3-5 \mathrm{~m} / \mathrm{s}(5,35)$ ] than the axonal velocities [normally $60-120 \mathrm{~m} / \mathrm{s}(41)$ ], the H-reflex latency is strongly influenced by the distance between the innervation zone and the recording electrodes. Assuming a muscle fiber conduction velocity of $4 \mathrm{~m} / \mathrm{s}$, a $1-\mathrm{cm}$ difference in electrode position over the muscle would change the measured latency by $2.5 \mathrm{~ms}$.

Due to the short distance between the stimulation and recording electrodes, an overlap between the stimulation artefact and the $\mathrm{M}$-wave occurred. Therefore, the M-wave and any potential influence of stimulation frequency on its amplitude could not be analyzed. However, at stimulation intensities evoking H-reflex amplitudes below 50\% of the maximum amplitude (which were the primary focus of this study), the M-wave was typically not present. This has been consistently observed across many muscles (75), including the extensor carpi radialis longus $(68,84)$. Furthermore, as the motor axon membrane potential repolarizes almost instantaneously after subthreshold current pulses (62), amplification of the response due to temporal summation was unlikely to have taken place in the motor axons. It should, however, be noted that, if some motor axons were recruited, they were likely to stay recruited due to their supernormal excitability when stimulated at 100 $\mathrm{Hz}$ (47). For these reasons, it was assumed that no or only a small degree of direct motor axon stimulation (M-wave) occurred during the experiments, and that the induced muscle activation was almost exclusively evoked via afferent pathways.

The results of this study indicate that the nonphysiological properties of the contractions elicited by classic NMES, i.e., nonordered recruitment order, perfect synchronization, and highly uniform discharge rates, could be partly overcome by activating the MNs indirectly via high-frequency stimulation of afferent fibers at a constant stimulation current that is below the motor axon rheobase current. Whether or not size-ordered recruitment can be achieved depends on the strength by which the Ia fibers project to the MN pool (Fig. 4), and it should further be noted that significant recruitment likely requires that a single-stimulus H-reflex of a certain magnitude can be elicited. While the present study serves to establish the basic feasibility of the approach and its physiological foundation, the functional applicability remains to be investigated. For example, the obvious extension of the proposed concept is the gradation of muscle force by modulation of the stimulation frequency. The range of forces that can be obtained with this approach, the sensitivity of force gradation, and the degree of the resulting muscle fatigue need to be characterized in detail in future work.

In conclusion, the simulation and experimental results of this study strongly support the working hypothesis that stimulation of afferent fibers at a sufficiently high frequency (in this case $100 \mathrm{~Hz}$ ) evokes temporal summation of EPSPs in MNs, amplifying the magnitude of their response to each stimulus and recruiting different groups of MNs in response to each individual stimulus. These findings suggest a potential for recruiting substantial numbers of MNs and evoking from them activity patterns more similar to physiological activation than what can be obtained using classical NMES.

\section{GRANTS}

This work was funded by the European Union Commission through the project NeuroTREMOR (contract no. 287739).

\section{DISCLOSURES}

No conflicts of interest, financial or otherwise, are declared by the author(s).

\section{AUTHOR CONTRIBUTIONS}

Author contributions: J.L.D., S.M., S.D., C.M.L., and D.F. conception and design of research; J.L.D. and S.M. performed experiments; J.L.D. analyzed data; J.L.D., S.M., S.D., C.M.L., and D.F. interpreted results of experiments; J.L.D. prepared figures; J.L.D. and D.F. drafted manuscript; J.L.D., S.M., S.D., C.M.L., and D.F. edited and revised manuscript; J.L.D., S.M., S.D., C.M.L., and D.F. approved final version of manuscript.

\section{REFERENCES}

1. Aimonetti JM, Schmied A, Vedel JP, Pagni S. Ia presynaptic inhibition in human wrist extensor muscles: effects of motor task and cutaneous afferent activity. J Physiol (Paris) 93: 395-401, 1999. 
2. Aimonetti JM, Vedel JP, Schmied A, Pagni S. Mechanical cutaneous stimulation alters Ia presynaptic inhibition in human wrist extensor muscles: a single motor unit study. J Physiol 522: 137-145, 2000.

3. Aimonetti JM, Vedel JP, Schmied A, Pagni S. Task dependence of Ia presynaptic inhibition in human wrist extensor muscles: a single motor unit study. Clin Neurophysiol 111: 1165-1174, 2000.

4. Aimonetti JM, Vedel JP, Schmied A, Pagni S. Distribution of presynaptic inhibition on type-identified motoneurones in the extensor carpi radialis pool in man. $J$ Physiol 522: 125-135, 2000.

5. Andreassen S, Arendt-Nielsen L. Muscle fibre conduction velocity in motor units of the human anterior tibial muscle: a new size principle parameter. J Physiol 391: 561-571, 1987.

6. Ashby P, Zilm D. Characteristics of postsynaptic potentials produced in single human motoneurons by homonymous group 1 volleys. Exp Brain Res 47: 41-48, 1982.

7. Awiszus F, Feistner H. The relationship between estimates of Ia-EPSP amplitude and conduction velocity in human soleus motoneurons. Exp Brain Res 95: 365-370, 1993.

8. Barret JN, Crill WE. Specific membrane resistivity of dye-injected cat motoneurons. Brain Res 28: 556-561, 1971.

9. Baudry S, Maerz AH, Enoka RM. Presynaptic modulation of Ia afferents in young and old adults when performing force and position control. J Neurophysiol 103: 623-631, 2010.

10. Bergquist AJ, Clair JM, Collins DF. Motor unit recruitment when neuromuscular electrical stimulation is applied over a nerve trunk compared with a muscle belly: triceps surae. J Appl Physiol 110: 627-637, 2011.

11. Bergquist AJ, Wiest MJ, Collins DF. Motor unit recruitment when neuromuscular electrical stimulation is applied over a nerve trunk compared with a muscle belly: quadriceps femoris. J Appl Physiol 113: 79-89, 2012.

12. Bickel CS, Gregory CM, Dean JC. Motor unit recruitment during neuromuscular electrical stimulation: a critical appraisal. Eur J Appl Physiol 111: 2399-2407, 2011.

13. Binboga E, Turker KS. Compound group I excitatory input is differentially distributed to human soleus motoneurons. Clin Neurophysiol 123 2192-2199, 2012.

14. Blouin JS, Walsh LD, Nickolls P, Gandevia SC. High-frequency submaximal stimulation over muscle evokes centrally generated forces in human upper limb skeletal muscles. $J$ Appl Physiol 106: 370-377, 2009.

15. Van Boxtel A. Differential effects of low-frequency depression, vibrationinduced inhibition, and posttetanic potentiation on H-reflexes and tendon jerks in the human soleus muscle. J Neurophysiol 55: 551-568, 1986.

16. Brown AG, Fyffe RE. Direct observations on the contacts made between Ia afferent fibres and alpha-motoneurones in the cat's lumbosacral spinal cord. J Physiol 313: 121-140, 1981.

17. Burke D, Gandevia SC, McKeon B. The afferent volleys responsible for spinal proprioceptive reflexes in man. $J$ Physiol 339: 535-552, 1983.

18. Burke D, Gandevia SC, McKeon B. Monosynaptic and oligosynaptic contributions to human ankle jerk and H-reflex. J Neurophysiol 52: 435-448, 1984.

19. Cisi RR, Kohn AF. Simulation system of spinal cord motor nuclei and associated nerves and muscles, in a Web-based architecture. J Comput Neurosci 25: 520-542, 2008.

20. Clair JM, Anderson-Rieid JM, Graham CM, Collins DF. Postactivation depression and recovery of reflex transmission during repetitive electrical stimulation of the human tibial nerve. J Neurophysiol 106: 184-192, 2011.

21. Clamann HP. Statistical analysis of motor unit firing patterns in a human skeletal muscle. Biophys J 9: 1233-1251, 1969.

22. Collins DF, Burke D, Gandevia SC. Large involuntary forces consistent with plateau-like behavior of human motoneurons. J Neurosci 21: 40594065, 2001.

23. Collins DF. Central contributions to contractions evoked by tetanic neuromuscular electrical stimulation. Exerc Sport Sci Rev 35: 102-109, 2007.

24. Cook W, Cangiano A. Presynaptic and postsynaptic inhibition of spinal motoneurons. J Neurophysiol 35: 389-403, 1972.

25. Crone C, Hultborn H, Kiehn O, Mazieres L, Wigstrom H. Maintained changes in motorneuronal excitability by short-lasting synaptic inputs in the decerebrate cat. J Physiol 405: 321-343, 1988

26. Cullheim S. Relations between cell body size, axon diameter and axon conduction velocity of cat sciatic alpha-motoneurons stained with horseradish peroxidase. Neurosci Lett 8: 17-20, 1978.
27. Curtis DR, Eccles JC. Synaptic action during and after repetitive stimulation. J Physiol 150: 374-398, 1960.

28. Day BL, Marsden CD, Obeso JA, Rothwell JC. Reciprocal inhibition between the muscles of the human forearm. J Physiol 349: 519-534, 1984.

29. Dean JC, Yates LM, Collins DF. Turning on the central contribution to contractions evoked by neuromuscular electrical stimulation. $J$ Appl Physiol 103: 170-176, 2007.

30. Delwaide PJ, Figiel C, Richelle C. Effects of postural changes of the upper limb on reflex transmission in the lower limb. J Neurol Neurosurg Psychiatry 40: 616-621, 1977.

31. Duchateau J, Balestra C, Carpentier A, Hainaut K. Reflex regulation during sustained and intermittent submaximal contractions in humans. $J$ Physiol 541: 959-967, 2002.

32. Eccles JC, Eccles RM, Lundberg A. Synaptic actions on motoneurones caused by impulses in Golgi tendon organ afferents. J Physiol 138: 227-252, 1957.

33. Eccles JC, Krnjevic K. Presynaptic changes associated with post-tetanic potentiation in the spinal cord. J Physiol 149: 274-287, 1959.

34. Enoka RM, Fuglevand AJ. Motor unit physiology: some unresolved issues. Muscle Nerve 24: 4-17, 2001

35. Farina D, Pozzo M, Merlo E, Bottin A, Merletti R. Assessment of average muscle fiber conduction velocity from surface EMG signals during fatiguing dynamic contractions. IEEE Trans Biomed Eng 51: 1383-1393, 2004.

36. Finkel AS, Redman SJ. The synaptic current evoked in cat spinal motoneurones by impulses in single group 1a axons. $J$ Physiol 342: 615-632, 1983

37. Fuglevand AJ, Winter DA, Patla AE. Models of recruitment and rate coding organization in motor-unit pools. J Neurophysiol 70: 2470-2488, 1993.

38. Gassel MM, Ott KH. Local sign and late effects on motoneuron excitability of cutaneous stimulation in man. Brain 93: 95-106, 1970.

39. Grillon C, Zarifian E. Hoffmann reflex variations produced by task demand characteristics. Physiol Behav 34: 213-216, 1985.

40. Hallbeck MS. Flexion and extension forces generated by wrist-dedicated muscles over the range of motion. Appl Ergon 25: 379-385, 1994.

41. Heckman CJ, Binder MD. Analysis of effective synaptic currents generated by homonymous Ia afferent fibers in motoneurons of the cat. $J$ Neurophysiol 60: 1946-1966, 1988.

42. Hennings K, Arendt-Nielsen L, Andersen O. Orderly activation of human motor neurons using electrical ramp prepulses. Clin Neurophysiol 116: 597-604, 2005.

43. Hultborn H. State-dependent modulation of sensory feedback. J Physiol 533: 5-13, 2001

44. Hunt CC. Relation of function to diameter in afferent fibers of muscle nerves. J Gen Physiol 38: 117-131, 1954.

45. Jami L. Golgi tendon organs in mammalian skeletal muscle: functional properties and central actions. Physiol Rev 72: 623-666, 1992.

46. Katz R, Morin C, Pierrot-Deseilligny E, Hibino R. Conditioning of $\mathrm{H}$ reflex by a preceding subthreshold tendon reflex stimulus. J Neurol Neurosurg Psychiatry 40: 575-580, 1977.

47. Kiernan MC, Mogyoros I, Burke D. Differences in the recovery of excitability in sensory and motor axons of human median nerve. Brain 119: 1099-1105, 1996.

48. Kitago T, Mazzocchio R, Liuzzi G, Cohen LG. Modulation of H-reflex excitability by tetanic stimulation. Clin Neurophysiol 115: 858-861, 2004.

49. Klakowicz PM, Baldwin ER, Collins DF. Contribution of M-waves and H-reflexes to contractions evoked by tetanic nerve stimulation in humans. J Neurophysiol 96: 1293-1302, 2006.

50. Knikou M. The H-reflex as a probe: pathways and pitfalls. $J$ Neurosci Methods 171: 1-12, 2008

51. Kuno M, Miyahara JT. Non-linear summation of unit synaptic potentials in spinal motoneurones of the cat. $J$ Physiol 201: 465-477, 1969.

52. Lagerquist $\mathbf{O}$, Collins DF. Influence of stimulus pulse width on $\mathbf{M}$ waves, H-reflexes, and torque during tetanic low-intensity neuromuscular stimulation. Muscle Nerve 42: 886-893, 2010.

53. Lloyd DP. Temporal summation in rhythmically active monosynaptic reflex pathways. J Gen Physiol 40: 427-434, 1957.

54. De Luca CJ, Foley PJ, Erim Z. Motor unit control properties in constant-force isometric contractions. J Neurophysiol 76: 1503-1516, 1996.

55. Macefield VG, Gandevia SC, Bigland-Ritchie B, Gorman RB, Burke D. The firing rates of human motoneurones voluntarily activated in the absence of muscle afferent feedback. J Physiol 471: 429-443, 1993. 
56. Maffiuletti NA. Physiological and methodological considerations for the use of neuromuscular electrical stimulation. Eur J Appl Physiol 110: 223-234, 2010.

57. Malesević NM, Popović LZ, Schwirtlich L, Popović DB. Distributed low-frequency functional electrical stimulation delays muscle fatigue compared to conventional stimulation. Muscle Nerve 42: 556-562, 2010.

58. Marchand-Pauvert V, Mazavet D, Nielsen J, Petersen N, PierrotDeseilligny E. Distribution of non-monosynaptic excitation to early and late recruited units in human forearm muscles. Exp Brain Res 134: 274-278, 2000.

59. Marchand-Pauvert V, Nicolas G, Burke D, Pierrot-Deseilligny E. Suppression of the H reflex in humans by disynaptic autogenetic inhibitory pathways activated by the test volley. J Physiol 542: 963-976, 2002.

60. Matthews PB. Relationship of firing intervals of human motor units to the trajectory of post-spike after-hyperpolarization and synaptic noise. $J$ Physiol 492: 597-628, 1996.

61. Mazzocchio R, Rothwell JC, Rossi A. Distribution of Ia effects onto human hand muscle motoneurones as revealed using an $\mathrm{H}$ reflex technique. J Physiol 489: 263-273, 1995.

62. McNeal DR. Analysis of a model for excitation of myelinated nerve. IEEE Trans Biomed Eng 23: 329-337, 1976.

63. Mendell LM, Henneman E. Terminals of single Ia fibers: location, density, and distribution within a pool of 300 homonymous motoneurons. J Neurophysiol 34: 171-187, 1971.

64. Miles TS, Le TH, Türker KS. Biphasic inhibitory responses and their IPSPs evoked by tibial nerve stimulation in human soleus motor neurones. Exp Brain Res 77: 637-645, 1989.

65. Misiaszek JE. The H-reflex as a tool in neurophysiology: its limitations and uses in understanding nervous system function. Muscle Nerve 28: 144-160, 2003.

66. Morita H, Baumgarten J, Petersen N, Christensen L, Nielsen J. Recruitment of extensor-carpi-radialis motor units by transcranial magnetic stimulation and radial-nerve stimulation in human subjects. Exp Brain Res 128: 557-562, 1999.

67. Moritz CT, Barry BK, Pascoe MA, Enoka RM. Discharge rate variability influences the variation in force fluctuations across the working range of a hand muscle. J Neurophysiol 93: 2449-2459, 2005.

68. Nafati G, Rossi-Durand C, Schmied A. Proprioceptive control of human wrist extensor motor units during an attention-demanding task. Brain Res 1018: 208-220, 2004.

69. Negro F, Farina D. Decorrelation of cortical inputs and motoneuron output. J Neurophysiol 106: 2688-2697, 2011.

70. Nelson SG, Mendell LM. Projection of single knee flexor Ia fibers to homonymous and heteronymous motoneurons. J Neurophysiol 41: $778-$ 787, 1978.

71. Pierrot-Deseilligny E, Bussel B, Held JP, Katz R. Excitability of human motoneurones after discharge in a conditioning reflex. Electroencephalogr Clin Neurophysiol 40: 279-287, 1976.
72. Powers RK, Binder MD. Distribution of oligosynaptic group I input to the cat medial gastrocnemius motoneuron pool. J Neurophysiol 53: $497-$ $517,1985$.

73. Rubinstein JT, Spelman FA. Analytical theory for extracellular electrical stimulation of nerve with focal electrodes. I. Passive unmyelinated axon. Biophys J 54: 975-981, 1988.

74. Rudomin P, Burke RE, Núñez R, Madrid J, Dutton H. Control by preynaptic correlation: a mechanism affecting information transmission from Ia fibers to motoneurons. J Neurophysiol 38: 267-284, 1975.

75. Schieppati M. The Hoffmann reflex: a means of assessing spinal reflex excitability and its descending control in man. Prog Neurobiol 28: 345376, 1987.

76. Schmied A, Morin D, Vedel JP, Pagni S. The "size principle" and synaptic effectiveness of muscle afferent projections to human extensor carpi radialis motoneurones during wrist extension. Exp Brain Res 113: 214-229, 1997.

77. Schmied A, Vedel JP, Calvin-Figuiere S, Rossi-Durand C, Pagni S. Task-dependence of muscle afferent monosynaptic inputs to human extensor carpi radialis motoneurones. Electroencephalogr Clin Neurophysiol 105: 220-234, 1997.

78. Scutter SD, Türker KS. Muscle spindle afferent input to motoneurons in human masseter. J Neurophysiol 82: 505-507, 1999.

79. Semmler JG, Turker KS. Compound group I excitatory input is differentially distributed to motoneurons of the human tibialis anterior. Neurosci Lett 178: 206-210, 1994.

80. Táboríková H, Sax DS. Conditioning of H-reflexes by a preceding subthreshold H-reflex stimulus. Brain 92: 203-212, 1969.

81. Thompson AK, Wolpaw JR. Operant conditioning of spinal reflexes: from basic science to clinical therapy. Front Integr Neurosci 8: 25, 2014.

82. Uysal H, Kizilay F, Inel SE, Ozen H, Pek G. Medium-latency reflex response elicited from the flexor carpi radialis by radial nerve stimulation. Exp Brain Res 217: 223-235, 2012.

83. Veale JL, Mark RF, Rees S. Differential sensitivity of motor and sensory fibres in human ulnar nerve. J Neurol Neurosurg Psychiatry 36: 78-86, 1973.

84. Wargon I, Lamy JC, Baret M, Ghanim Z, Aymard C, Pénicaud A, Katz R. The disynaptic group I inhibition between wrist flexor and extensor muscles revisited in humans. Exp Brain Res 168: 203-217, 2006.

85. Watt DG, Stauffer EK, Taylor A, Reinking RM, Stuart DG. Analysis of muscle receptor connections by spike-triggered averaging. 1. Spindle primary and tendon organ afferents. J Neurophysiol 39: 1375-1392, 1976.

86. Wood L, Nicol DJ, Thulin CE. The effects of skin brushing on H reflex amplitude in normal human subjects. Exp Physiol 83: 175-183, 1998.

87. Zengel JE, Reid SA, Sypert GW, Munson JB. Presynaptic inhibition, EPSP amplitude, and motor-unit type in triceps surae motoneurons in the cat. J Neurophysiol 49: 922-931, 1983. 